$\dagger$ Present address: Department of Physics, Washington State University, Pullman, Wash. 99163.

$¥$ Present address: Wave Propagation Laboratory, ESSA, Boulder, Colo. 80302.

\$ Visiting Member, Joint Institute for Laboratory Astrophysics, University of Colorado.

I G. Gräff, W. Paul, and C. Schlier, Z. Physik 153, 38 (1958); W. Drechsler and G. Gräff, ibid. 163, 165 (1961); R. von Boeckh, G. Gräff, and R. Ley, ibid. 179, 285 (1964).

2 R. H. Hammerle, J. T. Dickinson, R. G. Van Ausdal, D. A. Stephenson, and J. C. Zorn, J. Chem. Phys. 50, 2086 (1969).

${ }^{3}$ D. A. Stephenson, J. T. Dickinson, and J. C. Zorn, J. Chem. Phys. 53, 1529 (1970), following article.

${ }^{4}$ A preliminary report of this work has been given: J. T. Dickinson, D. A. Stephenson, and J. C. Zorn, Bull. Am. Phys. Soc. 13, 21 (1968).

${ }_{5}$ J. T. Dickinson, Ph.D. thesis, University of Michigan, Ann Arbor, Mich, University Microfilms, Inc., Ann Arbor, Mich., 1968.

${ }^{6}$ J. C. Zorn, T. C. English, J. T. Dickinson, and D. A. Stephenson, J. Chem. Phys. 45, 3731 (1966).

${ }^{7}$ H. G. Fitzky, Z. Physik 151, 351 (1958).
${ }^{8}$ H. G. Bennewitz, W. Paul, and C. Schlier, Z. Physik 141, 6 (1955).

- A nuclear magnetic octupole interaction has been observed in atomic bromine by H. Brown and J. G. King, Phys. Rev. 142, 53 (1966).

${ }^{10}$ R. L. White, Rev. Mod. Phys. 27, 276 (1955); C. Schlier, Fortschr. Physik 9, 455 (1961).

11 H. J. Zeiger and D. I. Bolef, Phys. Rev. 85, 788 (1952).

${ }^{12} \mathrm{C}$. H. Townes, "Determination of Nuclear Quadrupole Moments," in Handbuch der Physik, edited by S. Flügge (Springer, Berlin, 1958), Vol. 38, Pt. 1.

${ }^{13}$ G. R. Gunther-Mohr, S. Geschwind, and C. H. Townes, Phys. Rev. 81, 289 (1951); P. A. Bonczyk and V. H. Hughes, ibid. 161,15 (1967).

${ }_{14}$ H. M. Foley, Phys. Rev. 72, 504 (1947).

${ }_{15}$ For TlBr, the ratio of $a_{79} / a_{81}$ was used for comparison, where $a$ is the first coefficient in Eq. (2). The ratio obtained for TIBr is $a_{79} / a_{81}=1.1970530(20)$.

${ }_{16}$ H. H. Brown and J. G. King, Phys. Rev. 142, 53 (1966). The ratio of quadrupole interaction constants they obtain is $b_{79} / b_{81}=1.1970568(15)$.

\title{
Hyperfine Structure of Thallium Iodide and an Upper Limit for the Electric Hexadecapole Moment of the Iodine Nucleus*
}

\author{
David A. Stephenson, $\uparrow$ J. Thomas Dicktnson, $\ddagger$ and Jens C. Zorn $\S$ \\ Randall Laboratory of Physics, University of Michigan, Ann Arbor, Michigan 48104
}

(Received 10 March 1970)

\begin{abstract}
The hyperfine structure of thallium iodide has been studied in the $J=3$ and $J=4$ rotational states in an attempt to observe a nuclear electric hexadecapole effect. This study was carried out on a high-resolution molecular-beam electric resonance spectrometer at very weak electric and magnetic fields. The molecule is well described by the usual five hyperfine interaction constants which are (in kilohertz)

\begin{tabular}{|c|c|c|}
\hline & $\left.{ }^{205} \mathrm{~T}\right]^{127} \mathrm{I}$ & ${ }^{203} \mathrm{~T}^{12 \pi} \mathrm{I}$ \\
\hline$e q Q$ & $-438916.3 \pm 0.5$ & $-438917.3 \pm 1.0$ \\
\hline$c_{1}$ & $3.05 \pm 0.05$ & $3.09 \pm 0.1$ \\
\hline$c_{2}$ & $34.65 \pm 0.15$ & $34.36 \pm 0.3$ \\
\hline$c_{3}$ & $-2.48 \pm 0.1$ & $-2.59 \pm 0.2$ \\
\hline$c_{1}$ & $-6.67 \pm 0.05$ & $-6.57 \pm 0.1$ \\
\hline
\end{tabular}
\end{abstract}

No evidence for a hexadecapole interaction constant as large as $500 \mathrm{~Hz}$ was found; this result is interpreted as setting an upper limit of $\sim 10^{-47} \mathrm{~cm}^{4}$ for the nuclear electric hexadecapole moment of ${ }^{127} \mathrm{I}$.

\section{INTRODUCTION}

We have measured the radio-frequency spectrum of the thallium iodide molecules $\left.{ }^{203} \mathrm{~T}\right|^{127} \mathrm{I}$ and ${ }^{205} \mathrm{~T} \mathrm{l}^{127} \mathrm{I}$ with a molecular-beam electric resonance (MBER) spectrometer. We had two motivations for this work. First, this experiment completes a series of studies on the hyperfine structure of the thallium halides. Second, the TII molecule appeared to offer a good opportunity to identify and measure a nuclear electric hexadecapole (24-pole) interaction. The iodine nucleus has a large electric quadrupole moment ${ }^{1}$ and an observed magnetic octupole moment, ${ }^{2}$ both of which suggest the existence of higher-order moments; moreover the spin of iodine $\left(\frac{5}{2}\right)$ is large enough to allow the nucleus to exhibit a hexadecapole interaction. In addition, we know that the quadrupole hfs of $\mathrm{TlCl}$ and $\mathrm{TlBr}$, in which the halogen nuclear spin is less than 2 , is very well understood, ${ }^{3,4}$ so any deviations from quadrupole hfs intervals in TII might be attributed with confidence to the interaction of a higher-order nuclear moment.

\section{EXPERIMENT}

The important features of the MBER spectrometer used for these measurements have been described elsewhere. ${ }^{5,6}$ In addition to working with single resonances, we used a microwave rf double-resonance technique to identify some of the observed radiofrequency transitions. By observing the intensity of a given rf transition (which occurs within a single rotational state) as different rotational transitions were induced with a microwave $C$ field, we were able to determine which rotational state was involved in the rf transition.

As pointed out in the preceding paper, ${ }^{4}$ electric 
TABLE I. Hyperfine coupling constants for thallium iodide (in kilohertz).

\begin{tabular}{|c|c|c|c|c|c|}
\hline & $e q Q$ & $c_{1}$ & $c_{2}$ & $c_{3}$ & $c_{4}$ \\
\hline \multicolumn{6}{|c|}{${ }^{2055} \mathrm{Tl}^{127} \mathrm{I}$} \\
\hline $\begin{array}{l}v=0 \\
v=1 \\
v=2 \\
v=3\end{array}$ & $\begin{array}{ll}-438 & 916.3 \pm 0.5 \\
-440 & 509.8 \pm 1.0 \\
-442 & 095.0 \pm 3.0 \\
-443 & 674.0 \pm 4.0\end{array}$ & $\begin{array}{l}3.05 \pm 0.05 \\
3.06 \pm 0.1\end{array}$ & $\begin{array}{l}34.65 \pm 0.15 \\
34.60 \pm 0.3\end{array}$ & $\begin{array}{l}-2.48 \pm 0.1 \\
-2.42 \pm 0.2\end{array}$ & $\begin{array}{l}-6.67 \pm 0.05 \\
-6.69 \pm 0.1\end{array}$ \\
\hline \multicolumn{6}{|c|}{${ }^{203} \mathrm{~T}^{1227} \mathrm{I}$} \\
\hline $\begin{array}{l}\nu=0 \\
y=1 \\
v=2 \\
v=3\end{array}$ & $\begin{array}{l}-438917.3 \pm 1.0 \\
-440509.9 \pm 2.0 \\
-442099.0 \pm 4.0 \\
-443681.0 \pm 6.0\end{array}$ & $\begin{array}{l}3.09 \pm 0.1 \\
3.00 \pm 0.2\end{array}$ & $\begin{array}{l}34.36 \pm 0.3 \\
34.47 \pm 0.5\end{array}$ & $\begin{array}{l}-2.59 \pm 0.2 \\
-2.39 \pm 0.4\end{array}$ & $\begin{array}{l}-6.57 \pm 0.1 \\
-6.74 \pm 0.2\end{array}$ \\
\hline
\end{tabular}

focusing fields have a small aperture for the lowest rotational states of a molecule such as TII; our spectrometer provided adequate state selection only for the $J=3$ and $J=4$ states. This is not a serious handicap, for the numerous hyperfine energy levels in $J>1$ rotational states permit us to make a better test of the Hamiltonian than would be possible from the $J=1$ spectrum; moreover, it is only for $J>1$ states that a nuclear electric hexadecapole interaction can be observed.

About 90 rf spectral lines were observed in the $J=3$ and $J=4$ rotational states; useful results were obtained for the $v=0,1,2,3$ vibrational states. The most intense transition was the $39.441 \mathrm{MHz},\left(F_{1}=\frac{1}{2}\right.$, $F=1) \rightarrow\left(F_{1}=\frac{3}{2}, F=1\right)$ transition which occurs in the $J=3, v=0$ state of ${ }^{205} \mathrm{Tl}^{127} \mathrm{I}$. The full width at halfmaximum of the spectral lines was about $400 \mathrm{~Hz}$, which is expected from the transit time of the molecules through the $50-\mathrm{cm} C$ field.

\section{ANALYSIS OF SPECTRA}

\section{A. Hamiltonian}

Since the thallium nucleus in the $1 \Sigma$ TII molecule has spin $I_{2}=\frac{1}{2}$, the appropriate Hamiltonian is

$$
\begin{aligned}
H= & F_{v, J^{o p}}+Q^{(2)}: V^{(2)}+H^{(4)}: V^{(4)}+c_{1} I_{1} \cdot \mathrm{J}+c_{2} \mathrm{I}_{2} \cdot \mathrm{J} \\
+ & +c_{3} \mathrm{I}_{1} \cdot \mathbf{d}(\mathrm{J}) \cdot \mathrm{I}_{2}+c_{4}\left(\mathrm{I}_{1} \cdot \mathrm{I}_{2}\right)-\mu_{\bullet} \cdot \mathbf{E}-g_{J} \mu_{0} \mathrm{~J} \cdot \mathrm{H} \\
& -\mu_{N} \mathrm{H} \cdot\left(g_{1} \mathrm{I}_{1}+g_{2} \mathrm{I}_{2}\right) .
\end{aligned}
$$

The $F_{v, J^{\text {op }}}$ term is the usual operator for a vibrating rotor. The next two terms represent the electric quadrupole and electric hexadecapole interactions of the iodine nucleus. The terms with coefficients $c_{1}$ and $c_{2}$ contain the interaction of the nuclear magnetic moments with the magnetic field which arises from the rotation of the molecule. The sixth and seventh terms are operators for the tensor and scalar parts of the nuclear spin-spin interaction. The remaining terms represent the interaction of the molecule with external electric and magnetic fields. In this experiment the $C$ field is so weak that the contributions to the energy from these external field terms are negligible.

The magnetic octupole interaction is too small to show up in the hfs of $\mathrm{TlCl}$ and $\mathrm{TIBr}$ as observed ${ }^{3.4}$ with our spectrometer. Since the octupole moments of these halogen nuclei are comparable to that of iodine, ${ }^{2}$ we do not include an octupole term in the Hamiltonian used for interpretation of the TII spectrum.

\section{B. Matrix Elements}

Since we are dealing here with hyperfine structure in very weak fields and since the quadrupole interaction between $I_{1}$ and $\mathbf{J}$ is the dominant interaction, it makes sense to calculate matrix elements in the $\left|\left(I_{1} J\right) F_{1} I_{2} F M_{F}\right\rangle$ representation. We want agreement with spectra in which the uncertainty in the positions of the lines is less than $100 \mathrm{~Hz}$, so for thallium iodide, in which the quadrupole interaction ${ }^{7}(\sim 440$ $\mathrm{MHz})$ is comparable to the rotational constant $(\sim 815$ $\mathrm{MHz}$ ), we find ${ }^{8}$ that the calculations must be carried to fourth order in the quadrupole interaction of the iodine nucleus and must include contributions off diagonal in $F_{1}$ from the magnetic interactions of the thallium nucleus.

We use a contraction of two spherical tensors for the quadrupole interaction operator since the form of this operator which has been used for analysis of many previous MBER experiments does not give correct values of matrix elements which are off diagonal in $J$. The matrix elements for the quadrupole interaction, expressed with $3-j$ and $6-j$ symbols, ${ }^{9}$ are ${ }^{10}$

$$
\begin{aligned}
\left\langle\left[\left(I_{1} J\right) F_{1} I_{2}\right] F M_{F}\left|Q^{(2)}: V^{(2)}\right|\left[\left(I_{1} J^{\prime}\right) F_{1}^{\prime} I_{2}\right] F^{\prime} M_{F^{\prime}}\right\rangle & =\frac{1}{4}(e q Q)(-1)^{I_{1}+F_{1}}\left[\left(2 J^{\prime}+1\right)(2 J+1)\right]^{1 / 2} \\
& \times\left\{\begin{array}{ccc}
F_{1} & J^{\prime} & I_{1} \\
2 & I_{1} & J
\end{array}\right\}\left(\begin{array}{ccc}
J^{\prime} & 2 & J \\
0 & 0 & 0
\end{array}\right) /\left(\begin{array}{rrr}
I_{1} & 2 & I_{1} \\
-I_{1} & 0 & I_{1}
\end{array}\right) \delta_{F_{1} F_{1}} \delta_{F F^{\prime}} \delta_{M_{F} M_{F^{\prime}}} .
\end{aligned}
$$


By analogy one can define the electric hexadecapole interaction operator ${ }^{11}$ as a contraction of two fourth-rank spherical tensors; the matrix elements of this interaction are $^{12}$

$$
\begin{aligned}
\left\langle\left[\left(I_{1} J\right) F_{1} I_{2}\right] F M_{F}\left|Q^{(4)}: V^{(4)}\right|\left[\left(I_{1} J^{\prime}\right) F_{1}^{\prime} I_{2}\right] F^{\prime} M_{F^{\prime}}\right\rangle & =\frac{1}{8}(e h H)(-1)^{I_{1}+F_{1}}\left[(2 J+1)\left(2 J^{\prime}+1\right)\right]^{1 / 2} \\
& \times\left\{\begin{array}{ccc}
F_{1} & J^{\prime} & I_{1} \\
4 & I_{1} & J
\end{array}\right\}\left(\begin{array}{lll}
J^{\prime} & 4 & J \\
& & \\
0 & 0 & 0
\end{array}\right) /\left(\begin{array}{rrr}
I_{1} & 4 & I_{1} \\
-I_{1} & 0 & I_{1}
\end{array}\right) \delta_{F_{1} F_{1}} \delta_{F_{F}^{\prime}} \delta_{M F_{F} M_{F^{\prime}} \cdot}
\end{aligned}
$$

Here $h$ represents the expectation value of the fourth derivative of the electric potential at the site of the nucleus, and $e H$ is the hexadecapole moment of the nucleus. Only diagonal $\left(J=J^{\prime}\right)$ matrix elements of this interaction need be considered for our work since the hexadecapole energy is quite small.

The matrix elements for the magnetic hyperfine interactions, including the elements off diagonal in $F_{1}$, have been given by English. ${ }^{13}$

\section{RESULTS}

\section{A. Hyperfine Constants for TII}

Since more transitions were observed within the $J=4$ rotational state than within $J=3$, the results listed in Table I were obtained by doing a leastsquares adjustment of the hfs constants for a best match between the predictions of the Hamiltonian and the $J=4$ spectrum. We have also calculated the hfs constants from the $J=3$ spectrum alone; the resultant values of the constants agree with those given in Table I but the uncertainties are somewhat larger.

The hfs constants for the first two vibrational states were determined as described above; however, not enough transitions were observed within $v=2$ and $v=3$ to determine all the constants independently. Instead the magnetic interaction constants were assumed to be the same as in $v=1$, and $e q Q$ was then chosen to give the best fit to the observed spectrum. This procedure is justified because the change in the values of the magnetic interaction constants between $v=0$ and $v=1$ is less than the uncertainties in the values of these constants.

As was found in thallium bromide, ${ }^{4}$ the size of $e q Q$ for the halogen nucleus has a noticeable dependence on the thallium mass only when the molecule is in a $v>0$ state.

\section{B. Limit for the Nuclear Hexadecapole Moment of Iodine}

A very satisfactory fit between prediction and observation is obtained with $e h H$ set equal to zero An electric hexadecapole coupling constant larger than $500 \mathrm{~Hz}$ would not have escaped detection in these measurements; from this we can estimate an upper limit for the nuclear electric hexadecapole moment of iodine. If we assume that the electric field at the iodine nucleus comes from a unit point charge located at the thallium nucleus $2.8 \AA$ away, then the $500-\mathrm{Hz}$ limit on $e h H$ implies the iodine hexadecapole moment is less than $5 \times 10^{-45} \mathrm{~cm}^{4}$. However, as has been pointed out by Sternheimer, ${ }^{14}$ the point-charge model neglects antishielding effects and thus gives far too low a value for the fourth derivative of the electric field at the site of the nucleus. Sternheimer ${ }^{15}$ judged $10^{3}$ to be an upper limit for the hexadecapole antishielding factor in molecules like TII, so we believe the upper limit on the hexadecapole moment of iodine from this experiment is about $10^{-47} \mathrm{~cm}^{4}$.

This limit is of the same order as the size of the hexadecapole moment which was reported for the ${ }^{115} \mathrm{In}$ nucleus by Mahler, James, and Tantilla. ${ }^{16}$ In their experiment, ultrasonically induced $|\Delta m|=3$ transitions in an InAs crystal were interpreted as evidence for the interaction of the hexadecapole moment of the indium nucleus. Their results, as well as ours, depend strongly on the assumed value of the hexadecapole antishielding factor. We are presently using our MBER spectrometer to measure the radio-frequency spectrum of ${ }^{115} \mathrm{In}^{19} \mathrm{~F}$ to look for evidence of the indium hexadecapole moment in the hyperfine structure of the free molecule.

Effects of higher-order nuclear deformations have been observed ${ }^{17}$ in the scattering of alpha particles from rare-earth nuclei. Hendrie et al. ${ }^{17}$ point out, however, that it is difficult to establish a direct relationship between the deformation parameters obtained in their scattering experiments and the electric multipole moments of the nucleus since one cannot assume that the charge and nuclear fields have exactly the same shape. Moreover they remark that the relationship between the deformation parameters and the multipole moments is not unique.

\section{ACKNOWLEDGMENTS}

We would like to thank Professor Thomas C. English, Professor Carl Miller, Robert Hammerle, and Ray Van Ausdal for their contributions to this research.

\footnotetext{
I * This research was supported by the U.S. Atomic Energy Commission and by the Michigan Memorial Phoenix Project.

+ Present address: Wave Propagation Laboratory, ESSA, Boulder, Colo.

f Present address: Physics Department, Washington State University, Pullman, Wash.
} 
§ Visiting Member, Joint Institute for Laboratory Astrophysics, University of Colorado.

${ }^{1}$ C. H. Townes, "Determination of Nuclear Quadrupole Moments," in Handbuch der Physik, edited by S. Flügge (Springer, Berlin, 1958), Vol. 38, Pt. 1.

${ }^{2}$ V. Jaccarino, J. G. King, R. A. Satten, and H. H. Stroke, Phys. Rev. 94, 1798 (1954).

${ }_{3}$ R. H. Hammerle, J. T. Dickinson, R. G. Van Ausdal, D. A. Stephenson, and J. C. Zorn, J. Chem. Phys. 50, 2086 (1969).

${ }^{4}$ J. T. Dickinson, D. A. Stephenson, and J. C. Zorn, J. Chem. Phys. 53, 1525 (1970), preceding paper.

5 D. A. Stephenson, thesis, University of Michigan, Ann Arbor, Mich., 1968, University Microfilms, Ann Arbor, Mich., 1968; D. A. Stephenson, J. T. Dickinson, and J. C. Zorn, Bull. Am. Phys. Soc. 13, 21 (1968).

${ }_{6}^{6}$ J. C. Zorn, T. C. English, J. T. Dickinson, and D. A. Stephenson, J. Chem. Phys. 45, 3731 (1966).

${ }^{7} \mathrm{H}$. G. Fitzky, Z. Physik 151, 351 (1958).

${ }^{8}$ C. E. Miller, J. Mol. Spectry. 35, 278 (1970).

${ }^{9}$ A. R. Edmonds, Angular Momentum in Quantum Mechanics (Princeton U. P., Princeton, N. J., 1960), 2nd ed.
${ }^{10}$ H. P. Benz, A. Bauder, and Hs. H. Günthard, J. Mol. Spectry. $21,156(1966)$.

${ }^{11}$ T. C. Wang, Phys. Rev. 99, 566 (1955); K. K. Svidzinskii, Soviet Maser Research (Consultant's Bureau/Plenum, New York, 1964), pp. 88-148.

${ }^{12}$ C. E. Miller, Ph.D. dissertation, University of Michigan, Ann Arbor, Mich., 1967, University Microfilms, Ann. Arbor, Mich., 1967).

${ }^{13}$ T. C. English and J. C. Zorn, J. Chem. Phys. 47, 3896, (1967).

${ }^{14}$ R. M. Sternheimer, Phys. Rev. Letters 6, 190 (1961); Phys. Rev. 123, 870 (1961); 127, 812 (1962).

${ }_{15}$ We are indebted to R. M. Sternheimer for this private communication and for his suggestions regarding the interpretation of nuclear hexadecapole interactions.

${ }^{16}$ R. J. Mahler, L. W. James, and W. H. Tantilla, Phys. Rev. Letters 16, 259 (1966).

${ }^{17}$ D. L. Hendrie, N. K. Glendenning, B. G. Harvey, O. N. Jarvis, H. H. Duhm, J. Saudinos, and J. Mahoney, Phys. Letters 26B, 127 (1968); D. A. Bromley and J. Weneser, Comments Nucl. Particle Phys. 2, 55 (1968).

\title{
Hard-Square Solids at High Densities
}

\author{
A. L. Beyerlein, ${ }^{*}$ W. G. Rudd, $†$ Z. W. Salsburg, and M. Buynoski \\ Department of Chemistry, Rice University, Houston, Texas 77001
}

(Received 16 March 1970)

\begin{abstract}
An asymptotic expansion for the Helmholtz free energy $F_{N}$ of a system of $N$ hard squares of side $\sigma$ is obtained, with the result

$$
F_{N} / N k_{B} T \underset{\tau \rightarrow 1}{\simeq} 2 \ln (\lambda / \sigma)-2 \ln (\tau-1)+C+D(\tau-1)+E(\tau-1)^{2}+\cdots,
$$

where $k_{B}$ is Boltzmann's constant, $\lambda$ is the mean thermal de Broglie wavelength, and $\tau$ is the ratio of the system area to the close-packed area. A formal expression is derived for the constant $C$, while $D, E, \cdots$ are the coefficients in the expansion of $2 \ln \left(\tau^{1 / 2}+1\right)$ in powers of $(\tau-1)$. The modified cell-cluster technique is used to evaluate $C$, with the result $C=-0.26042240$.
\end{abstract}

\section{INTRODUCTION}

In recent years there has been a great deal of interest in the thermodynamic properties of rigid-particle systems, mainly because their simplicity makes it possible to study both the low-density fluid states and the high-density solidlike states in the neighborhood of the close-packed limit. Thus they offer the possibility of studying a solid-fluid phase transition which is believed to occur in rigid-disk and rigid-sphere systems. ${ }^{1}$

Progress toward the evaluation of the free energy $F_{N}$ of the high-density solid phase of $N \nu$-dimensional rigid particles came with the development of the following asymptotic expansion ${ }^{2,3}$ :

$$
\begin{array}{r}
F_{N} / N k_{B} T \underset{\tau \rightarrow 1}{\simeq} \nu \ln (\lambda / \sigma)-\nu \ln (\tau-1)+C+D(\tau-1) \\
+E(\tau-1)^{2}+\cdots,
\end{array}
$$

where $k_{B}$ is the Boltzmann constant, $\lambda$ is the mean thermal de Broglie wavelength $\left(h^{2} / 2 \pi m k_{B} T\right)^{1 / 2}$, and $\sigma$ is the particle diameter. The expansion parameter $(\tau-1)$ is defined in terms of the system volume $V$ as

$$
\tau=V / V_{0},
$$

where $V_{0}$ is the close-packed volume. The related asymptotic expansion for the pressure, in which the free-volume expression is the leading term, is suggested by molecular dynamics calculations on systems of hard disks and hard spheres. ${ }^{4}$ The validity of this asymptotic expansion has been proved for finite systems of rigid particles. $^{2,5}$ However, except for certain special models, ${ }^{5,6}$ rigorous proof in the thermodynamic limit $N \rightarrow \infty$ is lacking. Despite the lack of rigorous proof, we believe the expansion in Eq. (1) correctly predicts thermodynamic properties of hard-core systems in the neighborhood of the close-packed limit.

The free energy obtained by estimating $C$, $D$, etc., may be used in conjunction with molecular dynamics or Monte Carlo pressures of the fluid phase to estimate the location of a possible solid-fluid phase transition, both in pressure and in coexistent phase densities, ${ }^{3}$ by a Maxwell double tangent construction. This approach to locating the phase transition may be advantageous over integration of Monte Carlo or molecular dynamics pressures through the transition region where troublesome virial fluctuations occur.

The estimation of the coefficients in Eq. (1) has thus 